\title{
The Trichoscopic "Golf Club Set" Sign for Bullous Aplasia Cutis Congenita
}

\author{
Mario Cutrone ${ }^{a}$ Ramon Grimalt ${ }^{b}$

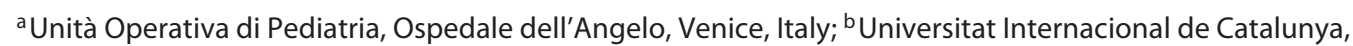 \\ Barcelona, Spain
}

\section{Established Facts}

- Aplasia cutis is a common condition.

- Bullous aplasia cutis is not so common.

- Trichoscopy may help in the diagnosis of aplasia cutis.

\section{Novel Insights}

- Bullous aplasia cutis might be confusing.

- A new trichoscopy sign ("golf club set") may help in achieving a diagnosis.

\section{Keywords}

Aplasia cutis - Dermoscopy - Bullous aplasia cutis · New trichoscopic sign - Trichoscopic "golf club set" sign

\begin{abstract}
Bullous aplasia cutis congenita (BACC) is a rather uncommon entity. The diagnosis can be quite tricky as the entity is not very frequent. Trichoscopy might in these cases be helpful to achieve the correct diagnosis. In this article, we describe for the first time a new sign for BACC that we believe can be useful to arrive at the correct diagnosis.
\end{abstract}

๑) 2018 S. Karger AG, Basel

\section{Introduction}

Aplasia cutis congenita (ACC) is a rare condition characterized by the absence of skin and sometimes other underlying structures, such as bone or dura. It can be a part of various syndromes and can be associated with multiple genetic diseases, malformation patterns, or a combination of all. In most cases, it is just a sporadic defect.

Bullous ACC (BACC) is a clinical subtype of AC, covered with a membranous surface. It has also been described as membranous or cystic AC. Clinically, it appears as a flat scar once the bullae have already reabsorbed. In some cases, there is an underlying neural tube defect. There are less than 20 cases reported in the literature [1] including the one discussed in this paper.

Trichoscopy can be useful to rule out other diagnoses, and we here propose a new trichoscopic sign that might help in diagnosing difficult cases.

\section{KARGER}

(C) 2018 S. Karger AG, Basel

E-Mail karger@karger.com

www.karger.com/sad
Dr. Ramon Grimalt

Facultat de Medicina i Ciències de la Salut

Universitat Internacional de Catalunya, Josep Trueta s/n

ES-08195 Sant Cugat del Vallès, Barcelona (Spain)

E-Mail rgrimalt@uic.es 
1

Fig. 1. Clinical image of our patient affected by bullous aplasia cutis.

Fig. 2. Trichoscopy revealed the presence of well-located hair distributed with the appearance of golf sticks under the translucent bullous membrane.
2

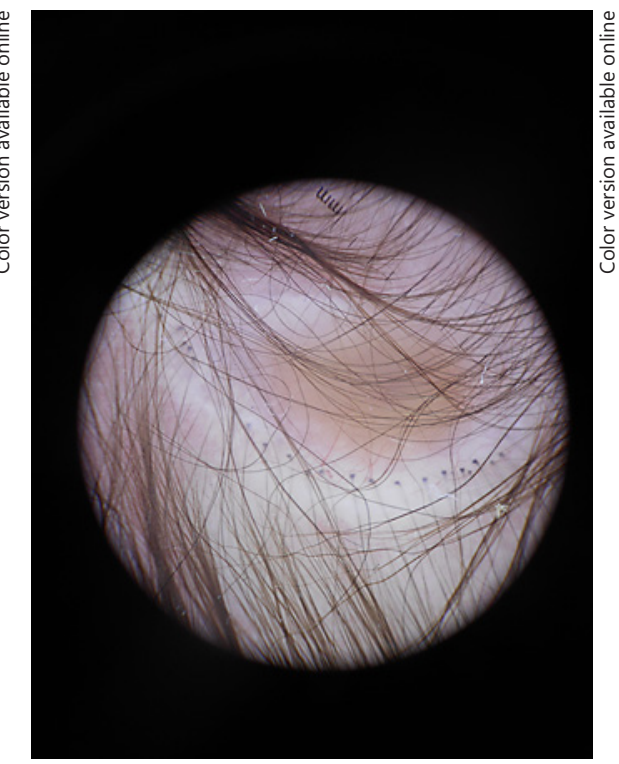

\section{Clinical Case}

A 2-day-old boy presented to one of us (M.C.) for a hairless patch, oval in shape and reddish in color, affecting the right parietal bone and present since birth. The first clinical impression was a type of AC (Fig. 1).

On a detailed clinical observation, the skin presented a welldelimitated border with the hair distributed in a rather peculiar collar-like way. Under the dermatoscope, we observed a distribution of hairs that were visible under the bullae with a highly characteristic aspect resembling a golf club set (Fig. 2). No skull bone and brain defects were found on ultrasonography.

\section{Discussion}

ACC is a congenital defect of the skin characterized by localized absence of the epidermis, dermis, and, at times, underlying structures, such as bone or dura [2]. It generally occurs on the scalp.

The etiology of AC is unknown. It might be caused by a combination of genetic factors, teratogens, compromised vasculature of the skin, and trauma. The increased incidence of lesions on the vertex is considered to be the result of a point of maximum tensile forces during rapid brain growth, inducing disruption of the overlying skin. Some authors have proposed that BACC is an incomplete type of neural tube defect and may be derived from a similar embryological defect. This is supported by several facts. First, the collar sign is a relatively specific marker for cranial neural tube closure defects. Second, the thin epithelial covering resembles that of encephaloceles and meningoceles both clinically and histologically [1].

Most cases appear to be sporadic, although some potential associations have been proposed: drugs, underlying embryologic malformations, infarction caused by rapid growth, among others [3]. The clinical picture varies from fissure-like ulcers with a granulating base to erosions, atrophic macules, or scars [4].

In a recent review, all the cases were located on the skull, most of them on the vertex or parietal scalp, ranging from 1 to 7 lesions, and frequently associated with bone defects [5]. Associated findings were hair collar sign, hydrocephaly, spasticity, epilepsy, cleft palate, primary optic nerve atrophy, meningeal arteriovenous fistula, corneal lipodermoid changes, cornea scleralization, nevus flammeus stain, and infantile hemangioma. Fujita et al. [6] reported 2 cases of AC, surrounded by a rim of hairs, without bony or neural defects: 1 case was associated with dense dermal melanocytosis and the other with nevus flammeus.

Drolet et al. [4] have proposed that BACC is an abortive form of a neural tube defect. The hair collar sign, regarded as a relatively specific marker for cranial neural tube closure defects, is frequently seen. Histologically, the thin epithelial covering observed resembles that of encephaloceles and meningoceles [5].

The diagnosis is clinical, and a biopsy is usually not needed. When performed, it shows an atrophic epidermis with loose fibrovascular stroma, edematous dermal stroma, or both [5]. A sonography of the lesion and a trans- 


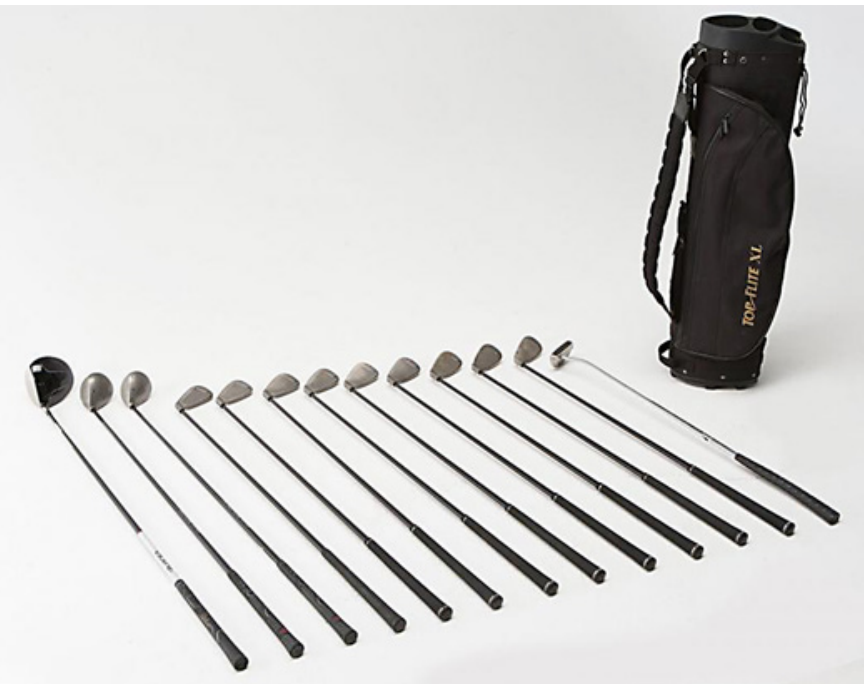

Fig. 3. A golf club set.

fontanellar sonography should be done to rule out skull defects or cerebral abnormalities [7]. BACC can be visualized on prenatal sonography as a smooth cystic lesion without flow [8]. Conservative therapy is the option of choice [3].
Lozano-Masdemont [9] recently published some trichoscopic findings in BACC, and Rakowska et al. [10] recently proposed the definition "hair bulbs arranged radially along hair-bearing margins" to describe a similar image. We believe that the new trichoscopic sign described in our article, "golf club set" (Fig. 3), does better illustrate the trichoscopic findings, might better help ruling out other diseases, and could be considered pathognomic of this entity.

Finally, Verzì et al. [11] in 2016 published an article with a very suggestive title describing the most common form of ACC (nonbullous form) with the term "starburst." We believe that this is a creative name that should be kept and that will help to differentiate our "golf club set" for BACC from "starburst" for ACC.

\section{Statement of Ethics}

Patient consent was obtained for pictures and eventual publication.

\section{Disclosure Statement}

The authors declare that they have no conflicts of interest.

\section{References}

1 Garcia-Romero MT, Narvóez-Rosales V, Hojyo-Tomoka MT: Bullous aplasia cutis congenita: case report and review of the literature. Indian J Dermatol 2011;56:337-338.

$\checkmark 2$ Frieden IJ: Aplasia cutis congenita: a clinical review and proposal for classification. J Am Acad Dermatol 1986;14:646-660.

3 Paller AS, Mancini AJ: Cutaneous disorders of the newborn; in Paller AS, Mancini AJ (eds): Hurwitz Clinical Pediatric Dermatology, ed 4. Philadelphia, Elsevier Saunders, 2011, pp 10-36.

4 Drolet B, Prendiville J, Golden J, Enjolras O, Esterly NB: "Membranous aplasia cutis" with hair collars. Congenital absence of skin or neuroectodermal defect? Arch Dermatol 1995; 131:1427-1431.
Colon-Fontanez F, Fallon Friedlander S, Newbury R, Eichenfield LF: Bullous aplasia cutis congenita. J Am Acad Dermatol 2003; 48(5 suppl):S95-S98.

6 Fujita Y, Yokota K, Akiyama M, Machino S, Inokuma $\mathrm{D}$, Arita $\mathrm{K}$, et al: Two cases of atypical membranous aplasia cutis with hair collar sign: one with dermal melanocytosis, and the other with naevus flammeus. Clin Exp Dermatol 2005;30:497-499.

7 Browning JC: Aplasia cutis congenita: approach to evaluation and management. Dermatol Ther 2013;26:439-444.
Jelin AC, Glenn OA, Strachowski L, Vargas JE: Membranous aplasia cutis congenita: a recognizable lesion on prenatal sonography. J Ultrasound Med 2009;28:1393-1396.

-9 Lozano-Masdemont B: A case of membranous aplasia cutis congenita and dermoscopic features. Int J Trichol 2017;9:33-34.

10 Rakowska A, Maj M, Zadurska M, et al: Trichoscopy of focal alopecia in children new trichoscopic findings: hair bulbs arranged radially along hair-bearing margins in aplasia cutis congenita. Skin Appendage Disord 2016;2:1-6.

11 Verzì AE, et al: Starburst hair follicles: a dermoscopic clue for aplasia cutis congenita. J Am Acad Dermatol 2016;75:e141-e142. 